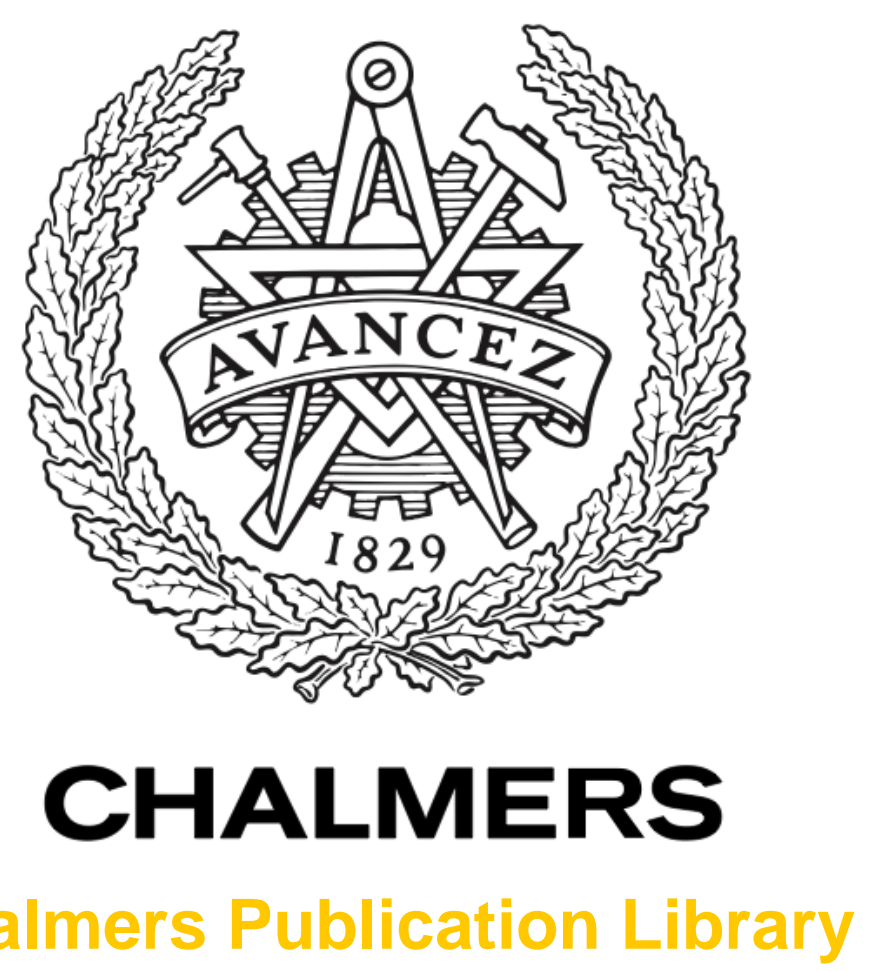

\title{
Dimensioning and Control of a Thermally Constrained Double Buffer Plug-in HEV Powertrain
}

This document has been downloaded from Chalmers Publication Library (CPL). It is the author's version of a work that was accepted for publication in:

51st IEEE Conference on Decision and Control, Maui, 10-13 December 2012 (ISSN: 0743-

1546)

Citation for the published paper:

Murgovski, N. ; Johannesson, L. ; Grauers, A. (2012) "Dimensioning and Control of a

Thermally Constrained Double Buffer Plug-in HEV Powertrain". 51 st IEEE Conference on

Decision and Control, Maui, 10-13 December 2012 pp. 6346-6351.

http://dx.doi.org/10.1109/CDC.2012.6426988

Downloaded from: http://publications.lib.chalmers.se/publication/164598

Notice: Changes introduced as a result of publishing processes such as copy-editing and formatting may not be reflected in this document. For a definitive version of this work, please refer to the published source. Please note that access to the published version might require a subscription.

Chalmers Publication Library (CPL) offers the possibility of retrieving research publications produced at Chalmers University of Technology. It covers all types of publications: articles, dissertations, licentiate theses, masters theses, conference papers, reports etc. Since 2006 it is the official tool for Chalmers official publication statistics. To ensure that Chalmers research results are disseminated as widely as possible, an Open Access Policy has been adopted.

The CPL service is administrated and maintained by Chalmers Library. 


\title{
Dimensioning and Control of a Thermally Constrained Double Buffer Plug-in HEV Powertrain
}

\author{
Nikolce Murgovski, Lars Johannesson, Anders Grauers and Jonas Sjöberg
}

\begin{abstract}
This paper describes modeling steps to enable fast evaluation of performance and cost effectiveness of a plugin hybrid electric vehicle. The paper also shows how convex optimization can be used to dimension the vehicle powertrain while simultaneously controlling the energy buffer power. The method allows for optimal control of powertrain components that are subject to thermal constraints.

The studied vehicle is a city bus driven along a perfectly known bus line. The bus is equipped with an engine-generator unit and an energy buffer consisting of an ultracapacitor and a battery. The engine generator unit and the energy buffer are modeled with quadratic power losses and are sized for two different charging scenarios. In the first scenario the bus can charge for a couple of seconds while standing still at bus stops, and in the second scenario the bus can charge for a couple of minutes before starting the route. In both scenarios, the ultracapacitor temperature is kept below a certain limit.
\end{abstract}

\section{INTRODUCTION}

Hybrid Electric Vehicles (HEVs) utilize one or more Electric Machines (EMs) and an energy buffer, typically a battery and/or an ultracapacitor, in addition to the Internal Combustion Engine (ICE). This gives them an additional degree of freedom that allows for a more efficient operation, due to: a possibility to recover braking energy by using the EMs as generators and storing the energy in the buffer; ability to shut down the ICE during idling and low load demands; possibility to run the ICE at more efficient load conditions while storing the excess energy in the buffer. See e.g. [1] for a detailed overview on hybrid vehicles.

Plug-in HEVs (PHEVs) have in addition a charging connector which allows them to draw electric energy from the grid. The PHEV's that are being considered in public transport are designed to charge conductively from fast-charge docking stations [2]-[4], or inductively from underground cables buried along sections of the bus line [5].

In order to be cost effective, the PHEV bus may need to include a downsized ICE and a carefully selected energy buffer to be able to drive a significant part of the bus line on electric power without a serious impact on vehicle performance. However dimensioning the PHEV powertrain, e.g. determining power rating and energy capacity of the energy buffer, is a difficult problem because it depends on charging infrastructure, drive patterns, topography along the bus line and on varying fuel and energy buffer prices [6].

Moreover, the energy efficiency of the powertrain depends on how well adapted the energy management strategy is to

The authors are with the Department of Signals and Systems, Charmers University of Technology, 41296 Gothenburg, Sweden nikolce.murgovski@chalmers.se, larsjo@chalmers.se, anders.grauers@chalmers.se, jonas.sjoberg@chalmers.se the bus line [7]. For PHEV city buses the energy management strategy decides the operating point of the ICE and thereby when and at which rate the energy buffer is to be discharged. When optimizing the PHEV public transportation system based on a dynamic model of the powertrain, a badly designed energy management may lead to a non-optimal size of the powertrain components [8]. Hence, to overcome this problem, both the size of the powertrain components and the energy management need to be optimized simultaneously.

Furthermore, city buses may also have tight daily schedules with short charging intervals, or the charging infrastructure might be sparsely distributed. This may require using the energy buffer under high duty cycles, thus increasing its operating temperature and possible degrading its performance. To prevent overheating, the energy buffer should be managed properly, and/or the cooling system should be dimensioned at the same time when sizing the buffer.

The problem of optimal sizing and control of HEVs is traditionally solved by Dynamic Programming [9] for which vast number of scientific articles are available [10][14]. The main advantage with DP is the capability to use nonlinear, non-convex models of the components consisting of continuous and integer (mixed-integer) optimization variables. However, a serious limitation of DP is that the computation time increases exponentially with the number of state variables [9]. As a consequence, the powertrain model is typically limited to only one or possibly two continuous state variables. Moreover, since DP operates by recursively solving a smaller subproblem for each time step, the second limitation of DP is that it is not possible to directly include the component sizing into the optimization. Instead, DP must be run in several loops to obtain the optimal control over a grid of component sizes.

In a more recent study [15] convex optimization has been used to simultaneously size a battery while optimally controlling a PHEV. The study considered approximated quadratic losses for the powertrain components and showed that the error due to the convexifying approximations is small as long as the battery open circuit voltage is nearly constant within the operating State of Charge (SOC) interval.

This paper is an extension of [15] and shows convexifying steps to allow for simultaneous dimensioning of three powertrain components while optimally controlling a PHEV bus with a series powertrain topology [1]. The components to be sized are engine-generator unit and energy buffer consisting of a battery with nearly linear voltage-SOC dependency and a thermally constrained ultracapacitor. The model dynamics are described with three continuous states, 


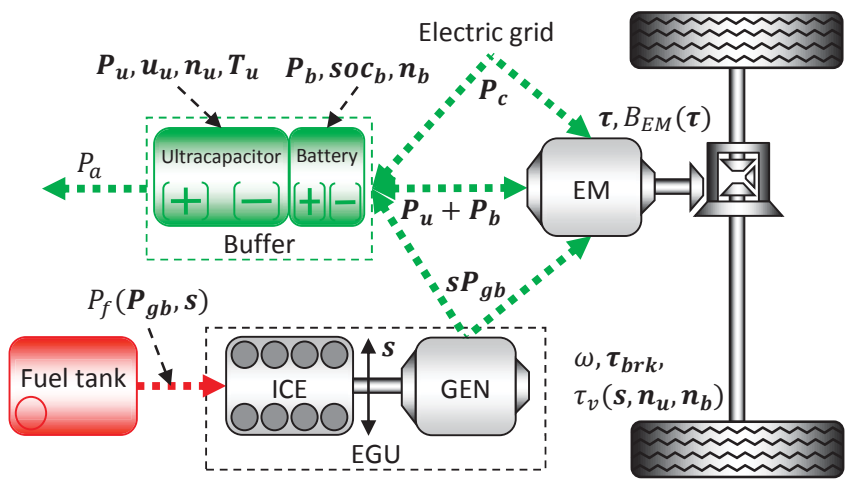

Fig. 1. Series PHEV powertrain model.

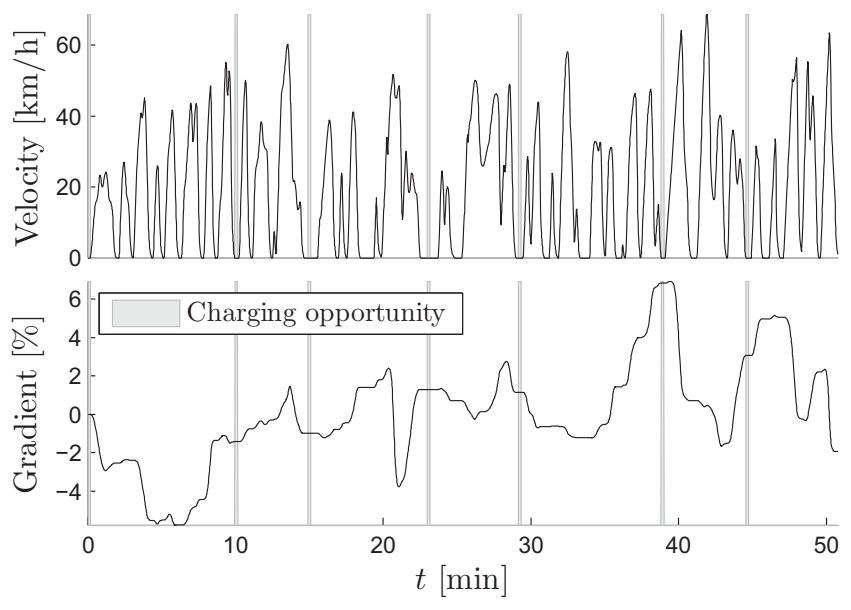

Fig. 2. Bus line model described by demanded velocity and road gradient. The shaded regions illustrate charging opportunities.

two for the battery and ultracapacitor SOC and one for the ultracapacitor temperature. The powertrain components are modeled with quadratic losses and the resulting convex problem is a semidefinite program [16].

The paper also gives an example showing how the optimal sizes of the components are affected for two different charging scenarios. In the first scenario the bus can charge for a couple of seconds while standing still at bus stops, and in the second scenario the bus can charge for a couple of minutes before starting the route.

The paper is outlined as follows: modeling details and problem formulation are discussed in Section II, III and IV, convex remodeling is discussed in Section V, an example of optimal dimensioning and control of a PHEV bus is given in Section VI, ending with conclusion in Section VII.

\section{BUS LINE AND POWERTRAIN MODEL}

The studied PHEV bus includes a powertrain in a series topology [1] that does not have a direct mechanical link between the ICE and the wheels, as in Fig. 1. Instead, the wheels are driven by an EM that receives energy from an ultracapacitor, a battery and/or an Engine-Generator Unit (EGU).

The bus is driven on a bus line described by a road gradient and demanded velocity which are known at each point of time (Fig. 2). The velocity and force demands from the bus line can be translated into an angular velocity $\omega(t)$ and torque

$$
\tau_{v}(\cdot)=\tau_{b}(t)+A_{1}(t) \boldsymbol{n}_{\boldsymbol{u}}+A_{2}(t) \boldsymbol{n}_{\boldsymbol{b}}+A_{3}(t) \boldsymbol{s}
$$

on the shaft between the EM and the differential. The number of ultracapacitor cells $\boldsymbol{n}_{\boldsymbol{u}}$, battery cells $\boldsymbol{n}_{\boldsymbol{b}}$ and the EGU size $s$ are decision variables (marked in bold), and (.) is used as a compact notation to identify a function of decision variables. The torque $\tau_{b}(t)$ of the vehicle without the weight of the energy buffer and EGU, and the time dependent terms $A_{j}(t)$ can be derived directly from the demanded acceleration and speed on the bus line and the known vehicle parameters, such as inertia, aerodynamic drag, rolling resistance, wheels radius, etc.

The EM, which delivers a torque $\tau(t)$, is designed to be able to meet the high torque demands. Moreover, the EM is also used to recuperate braking energy up to the point when either its torque limit $\tau_{\min }(\omega(t))$, or the buffer charging limit is reached, after which friction brakes are used to handle the remaining braking torque $\tau_{b r k}(t)$, i.e.

$$
\boldsymbol{\tau}(t)=\tau_{v}(\cdot)-\boldsymbol{\tau}_{\boldsymbol{b r k}}(t) .
$$

The powertrain electric path is described by a power balance

$$
\begin{aligned}
\boldsymbol{\tau}(t) \omega(t) & +B_{E M}(\cdot) \\
& =\boldsymbol{P}_{\boldsymbol{u}}(t)+\boldsymbol{P}_{\boldsymbol{b}}(t)+\boldsymbol{P}_{\boldsymbol{c}}(t)+\boldsymbol{s} \boldsymbol{P}_{\boldsymbol{g} \boldsymbol{b}}(t)-P_{a}
\end{aligned}
$$

that relates the EM electric power, left side of the equality, to the ultracapacitor power $\boldsymbol{P}_{\boldsymbol{u}}(t)$, battery power $\boldsymbol{P}_{\boldsymbol{b}}(t)$, grid charging power $\boldsymbol{P}_{\boldsymbol{c}}(t)$, EGU power $\boldsymbol{s} \boldsymbol{P}_{\boldsymbol{g} \boldsymbol{b}}(t)$ and power consumed by auxiliary devices $P_{a}$. The losses of the EM are modeled as a quadratic function on $\boldsymbol{\tau}(t)$

$$
B_{E M}(\cdot)=b_{0}(\omega(t)) \boldsymbol{\tau}^{2}(t)+b_{1}(\omega(t)) \boldsymbol{\tau}(t)+b_{2}(\omega(t))
$$

with speed dependent coefficients where $b_{j}(\omega(t)) \geq 0, j \in$ $\{0,2\}, \forall t \in\left[t_{0}, t_{f}\right]$.

The generator power, EGU losses and mass are assumed to scale linearly with the generator power $\boldsymbol{P}_{\boldsymbol{g} \boldsymbol{b}}(t)$, losses $B_{E G U b}(\cdot)$ and mass $m_{E G U b}$ of a baseline EGU model with maximum power of $P_{g b \max }=150 \mathrm{~kW}$. Then, the fuel power $P_{f}(\cdot)$ and mass $m_{E G U}$ of the scaled EGU can be expressed as

$$
\begin{aligned}
& P_{f}(\cdot)=\boldsymbol{s}\left(\boldsymbol{P}_{\boldsymbol{g} \boldsymbol{b}}(t)+B_{E G U b}(\cdot)\right) \\
& m_{E G U}=\boldsymbol{s} m_{E G U b}, \quad \boldsymbol{s} \in[0.5,1.5] .
\end{aligned}
$$

The losses of the baseline EGU are also modeled as quadratic

$$
B_{E G U b}(\cdot)=a_{0} \boldsymbol{P}_{\boldsymbol{g} \boldsymbol{b}}^{\mathbf{2}}(t)+a_{1} \boldsymbol{P}_{\boldsymbol{g} \boldsymbol{b}}(t)+a_{2} e(t)
$$

with $a_{j} \geq 0, j \in\{0,2\}$, where $e(t)$ is a binary variable that is needed to allow for zero fuel power, i.e. to remove the idling losses $a_{2}$ when the engine is off. The efficiencies of the EM and EGU are illustrated in Fig. 3, while details on the validity of using quadratic losses for these components can be found in [15].

The engine on/off control is decided using heuristics that turn the engine on if the power of the vehicle without the 

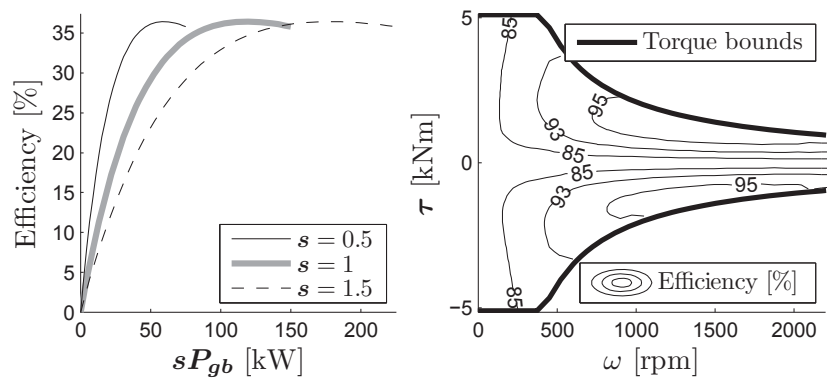

Fig. 3. Model of the EGU, left, and the EM, right. The figure illustrates the efficiency curves of the baseline EGU $(s=1)$, the smallest allowed EGU $(s=0.5)$, and the largest EGU $(s=1.5)$.

weight of the energy buffer and EGU exceeds a threshold $P_{o n}^{*}$, i.e.

$$
e(t)= \begin{cases}1, & \tau_{b}(t) \omega(t)>P_{o n}^{*} \\ 0, & \text { otherwise. }\end{cases}
$$

The optimal power threshold $P_{o n}^{*}$ is found by iteratively solving a convex optimization problem, described later in Section IV and V, for several values of $P_{\text {on }}$ within the power range of the vehicle. The detailed procedure can be found in [15], where it has been shown that these heuristics give small error to the global optimum.

The vehicle can charge from the electric grid while standing still at the terminal or at bus stops. It is assumed, for simplicity, that the chargers have equal maximum power $P_{c m a x}$ and constant efficiency $\eta$. The charging opportunities, shaded in Fig. 2, are indicated by a binary variable $c(t)$.

The losses of the power electronics are neglected, for simplicity, as they are typically much lower than the losses of the other powertrain components.

\section{ENERGY BUFFER MODEL}

The energy buffer consists of an ultracapacitor and a battery pack. Each pack consists of identical cells equally divided in parallel strings, with the strings consisting of cells connected in series.

\section{A. Ultracapacitor and battery cell}

The model of the buffer cells can be described through the following equations

$$
\begin{aligned}
& i_{j}(\cdot)=\frac{1}{2 R_{j}}\left(\boldsymbol{u}_{\boldsymbol{j}}(t)-\sqrt{\boldsymbol{u}_{\boldsymbol{j}}^{\mathbf{2}}(t)-\frac{4 R_{j} \boldsymbol{P}_{\boldsymbol{j}}(t)}{\boldsymbol{n}_{\boldsymbol{j}}}}\right) \\
& i_{j}(\cdot) \in\left[i_{\text {jmin }}, i_{\text {jmax }}\right] \\
& \boldsymbol{P}_{\boldsymbol{j}}(t) \leq \frac{\boldsymbol{u}_{\boldsymbol{j}}^{\boldsymbol{2}}(t) \boldsymbol{n}_{\boldsymbol{j}}}{4 R_{j}}, \quad j \in\{u, b\} \\
& \dot{\boldsymbol{u}}_{\boldsymbol{u}}(t)=-\frac{1}{C_{u}} i_{u}(\cdot) \\
& \boldsymbol{u}_{\boldsymbol{u}}(t) \in\left[0, u_{u \max }\right] \\
& \boldsymbol{u}_{\boldsymbol{u}}\left(t_{f}\right)=\boldsymbol{u}_{\boldsymbol{u}}\left(t_{0}\right) \\
& \boldsymbol{s}_{\boldsymbol{b}}(t)=-\frac{1}{Q_{b}} i_{b}(\cdot) \\
& \boldsymbol{s o c}_{\boldsymbol{b}}(t) \in\left[\operatorname{soc}_{b_{\text {min }}}, \text { soc }_{b \max }\right] \\
& \boldsymbol{s o c}_{\boldsymbol{b}}\left(t_{f}\right)=\boldsymbol{s o c}_{\boldsymbol{b}}\left(t_{0}\right)
\end{aligned}
$$

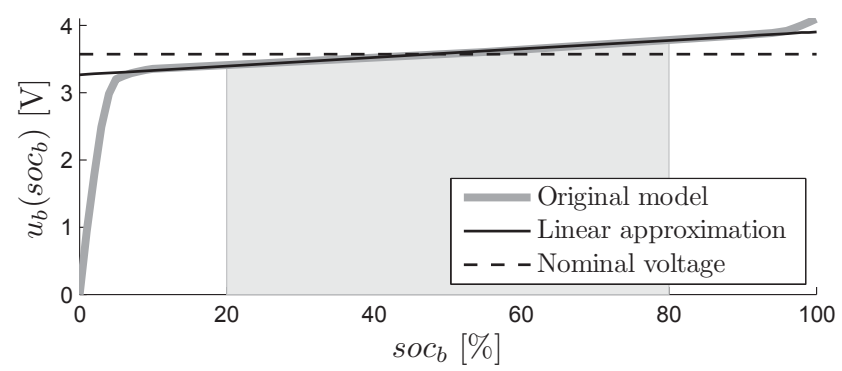

Fig. 4. Model of the battery open circuit voltage. The shaded region represents the allowed SOC range.

with $i_{j}(\cdot)$ and $\boldsymbol{u}_{j}(t)$ denoting the cell current and open circuit voltage, of the ultracapacitor $(j=u)$, and battery $(j=b)$. The cells are characterized by an inner resistance $R_{j}$, capacitance $C_{u}[\mathrm{~F}]$, or $Q_{b}[\mathrm{Ah}]$, current limits $i_{j \min }, i_{\text {jmax }}$ and maximum voltage $u_{u \max }$.

Note that (9a) has been derived from

$$
\boldsymbol{P}_{\boldsymbol{j}}(t)=\left(\boldsymbol{u}_{\boldsymbol{j}}(t) i_{j}(\cdot)-R_{j} i_{j}^{2}(\cdot)\right) \boldsymbol{n}_{\boldsymbol{j}}, \quad j \in\{u, b\}
$$

which shows that the power of each cell $\boldsymbol{P}_{\boldsymbol{j}}(t) / \boldsymbol{n}_{\boldsymbol{j}}$ is equal and does not depend on the configuration of cells (series/parallel), but rather on the total number of cells. Therefore, in the rest of the paper the sizing problem will focus only on determining the total number of cells in both the ultracapacitor and battery pack.

In the optimization $\boldsymbol{n}_{\boldsymbol{j}}$ has a real value that indicates the total pack capacity. It can be expected that rounding this variable to the nearest integer gives small error if results point to large number of cells. This will generally be the case if the cells are chosen small.

The decision variable describing ultracapacitor cell dynamics in (10a) is $\boldsymbol{u}_{\boldsymbol{u}}(t)$. The cell dynamics of the battery in (11a) are described by the SOC, $\boldsymbol{s o c}_{\boldsymbol{b}}(t)$, while the battery open circuit cell voltage is a nonlinear function of SOC, as shown in Fig. 4. The battery SOC range is limited by (11b), and charge sustain operation is required for both buffer packs by (10c) and (11c).

\section{B. Thermal state}

An objective of this study is to keep the ultracapacitor temperature $\boldsymbol{T}_{\boldsymbol{u}}(t)$ under a reasonable limit. The ultracapacitor is operated under natural convection cooling

$$
C_{T} R_{T} \dot{\boldsymbol{T}}_{\boldsymbol{u}}(t)=R_{T} R_{u} i_{u}^{2}(\cdot)+T_{a}-\boldsymbol{T}_{\boldsymbol{u}}(t)
$$

where it is required not only to keep the temperature below a certain limit $T_{\text {umax }}$,

$$
\boldsymbol{T}_{\boldsymbol{u}}(t) \leq T_{\text {umax }},
$$

but also to maintain a feasible temperature even when the bus line is to be driven many times under high ambient temperature $T_{a}$. This can be achieved by enforcing the optimization to sustain the initial ultracapacitor temperature at the end of the bus line,

$$
\boldsymbol{T}_{\boldsymbol{u}}\left(t_{0}\right)=\boldsymbol{T}_{\boldsymbol{u}}\left(t_{f}\right) .
$$


In the thermal model (12) it is assumed that all cells experience equal temperature and the only heat source is their own resistive loss. The thermal resistance and capacitance of the cells are denoted by $R_{T}$ and $C_{T}$, respectively.

\section{Problem Formulation}

The studied optimization problem is formulated to minimize a sum of operational cost for consumed fuel and electricity on the bus line and component cost for the EGU and the energy buffer. The costs are expressed in a single objective (15a) using coefficients $w_{f}, w_{c}$ in [currency/kWh], for fuel and electricity, respectively, and $w_{j}, j \in\{u, b, g\}$ in [currency], for $\{u, b, g\}=\{$ ultracapacitor, battery, EGU $\}$, respectively.

The optimization is subject to constraints invoked by the powertrain model forming the nonlinear optimization problem

$$
\begin{aligned}
& \operatorname{minimize} \int_{t_{0}}^{t_{f}} \begin{array}{c}
\left(w_{f} P_{f}(\cdot)+w_{c} \frac{\boldsymbol{P}_{\boldsymbol{c}}(t)}{\eta}\right) d t \\
\quad+w_{u} \boldsymbol{n}_{\boldsymbol{u}}+w_{b} \boldsymbol{n}_{\boldsymbol{b}}+w_{g} \boldsymbol{s}
\end{array} \\
& \forall t \in\left[t_{0}, t_{f}\right], \text { subject to }(9 \mathrm{~b})-(14) \text { and } \\
& \boldsymbol{\tau}(t) \geq \max \left\{\tau_{\min }(\omega(t)), \tau_{v}(\cdot)\right\} \\
& \boldsymbol{\tau}(t) \omega(t)+B_{E M}(\cdot) \\
& \quad \leq \boldsymbol{P}_{\boldsymbol{u}}(t)+\boldsymbol{P}_{\boldsymbol{b}}(t)+\boldsymbol{P}_{\boldsymbol{c}}(t)+\boldsymbol{s} \boldsymbol{P}_{\boldsymbol{g b}}(t)-P_{a} \\
& \boldsymbol{P}_{\boldsymbol{g} \boldsymbol{b}}(t) \in\left[0, P_{\text {gbmax }} e(t)\right] \\
& \boldsymbol{P}_{\boldsymbol{c}}(t) \in\left[0, \eta P_{c \max } c(t)\right] \\
& \boldsymbol{n}_{\boldsymbol{u}} \geq 0, \quad \boldsymbol{n}_{\boldsymbol{b}} \geq 0, \quad \boldsymbol{s} \in[0.5,1.5]
\end{aligned}
$$

with decision variables $\boldsymbol{P}_{\boldsymbol{u}}(t), \boldsymbol{P}_{\boldsymbol{b}}(t), \boldsymbol{P}_{\boldsymbol{c}}(t), \boldsymbol{P}_{\boldsymbol{g} \boldsymbol{b}}(t), \boldsymbol{\tau}(t)$, $\boldsymbol{u}_{\boldsymbol{u}}(t), \boldsymbol{s o c}_{\boldsymbol{b}}(t), \boldsymbol{T}_{\boldsymbol{u}}(t), \boldsymbol{n}_{\boldsymbol{u}}, \boldsymbol{n}_{\boldsymbol{b}}$ and $\boldsymbol{s}$.

The constraints (2) and (3) have been relaxed with inequalities in (15b) and (15c), respectively, and the braking torque has been taken out of the optimization problem. This does not change the optimal result, since at the optimum (15c) will hold with equality as otherwise energy will be wasted unnecessarily. Similarly, (15b) will also hold with equality, except during braking when the vehicle cannot recuperate all the braking energy. Then, the remaining energy is dissipated at the friction brakes and the optimal braking torque can be obtained directly from (2). See [15] for details.

The EGU price is assumed to follow an affine relation

$$
c_{E G U}=c_{0}+s c_{g}
$$

which together with known buffer cell prices $c_{u}, c_{b}$, can be used to obtain the coefficients $w_{j}$. It is assumed that the payment is divided in equal amounts over a period of $y=5$ years, with $p=5 \%$ yearly interest rate. Then, the equivalent components' cost related to the driven bus line is obtained by multiplying the length of the bus line with the components' price per kilometer, given the average travel distance $d=$ $80000 \mathrm{~km}$ in one year. This yields

$$
w_{j}=c_{j}\left(1+p \frac{y+1}{2}\right) \frac{\int_{t_{0}}^{t_{f}} v(t) d t}{y d}, \quad j \in\{u, b, g\}
$$

with $v(t)$ denoting the vehicle velocity demanded by the bus line.

\section{Convex Modeling}

This section gives a brief background on convex optimization and shows modeling steps to convexify the problem (15).

\section{A. Convex problem in a general form}

A convex problem can be written in the form

$$
\begin{array}{cl}
\operatorname{minimize} & f_{0}(\boldsymbol{x}) \\
\text { subject to } & f_{i}(\boldsymbol{x}) \leq 0, \quad i=1, \ldots, m \\
& h_{j}(\boldsymbol{x})=0, \quad j=1, \ldots, p \\
& \boldsymbol{x} \in \mathcal{X}
\end{array}
$$

where $\mathcal{X} \subseteq \mathbb{R}^{n}$ is a convex set, $f_{i}(\boldsymbol{x}), i=0, \ldots, m$ are convex functions and $h_{j}(\boldsymbol{x})$ are affine functions of the decision variables $\boldsymbol{x}$ [16]. The problem (15) does not comply with this definition and it needs to be remodeled. The modeling steps follow a disciplined methodology [16] where the convexity of complex functions is verified using operations that preserve convexity of elementary convex functions, e.g. affine $f(\boldsymbol{x})=q \boldsymbol{x}+r$, quadratic $f(\boldsymbol{x})=p \boldsymbol{x}^{2}+q \boldsymbol{x}+r$ with $p \geq 0$, quadratic-over-linear $f(\boldsymbol{x}, \boldsymbol{y})=\boldsymbol{x}^{2} / \boldsymbol{y}$ with $\boldsymbol{y}>0$, negative geometric mean $f(\boldsymbol{x}, \boldsymbol{y})=-\sqrt{\boldsymbol{x} \boldsymbol{y}}$ with $\boldsymbol{x} \geq 0, \boldsymbol{y} \geq 0$, etc.

\section{B. Convex EGU model}

The EGU can be modeled as convex by introducing a variable change $\boldsymbol{P}_{\boldsymbol{g}}(t)=\boldsymbol{s} \boldsymbol{P}_{\boldsymbol{g} \boldsymbol{b}}(t)$ that eliminates the nonconvex product of two variables. The change affects $P_{f}(\cdot)$ making the cost function (15a) convex. The constraint (15d) is also affected, but its convexity is preserved, yielding

$$
\begin{aligned}
& P_{f}(\cdot)=a_{0} \frac{\boldsymbol{P}_{\boldsymbol{g}}^{2}(t)}{\boldsymbol{s}}+\left(a_{1}+1\right) \boldsymbol{P}_{\boldsymbol{g}}(t)+\boldsymbol{s} a_{2} e(t) \\
& \boldsymbol{P}_{\boldsymbol{g}}(t) \in\left[0, \boldsymbol{s} P_{\text {gbmax }} e(t)\right] .
\end{aligned}
$$

\section{Convex ultracapacitor model}

The ultracapacitor can be modeled as convex by introducing a new variable $\boldsymbol{B}_{\boldsymbol{u}}(t)=R_{u} i_{u}^{2}(\cdot) \boldsymbol{n}_{\boldsymbol{u}}$ denoting pack losses, and performing a variable change

$$
\boldsymbol{E}_{\boldsymbol{u}}(t)=\frac{C_{u} \boldsymbol{u}_{\boldsymbol{u}}^{\mathbf{2}}(t) \boldsymbol{n}_{\boldsymbol{u}}}{2} \Rightarrow \dot{\boldsymbol{E}}_{\boldsymbol{u}}(t)=C_{u} \boldsymbol{n}_{\boldsymbol{u}} \boldsymbol{u}_{\boldsymbol{u}}(t) \dot{\boldsymbol{u}}_{\boldsymbol{u}}(t)
$$

that replaces cell voltage with pack energy. The pack losses then become

$$
\begin{aligned}
\boldsymbol{B}_{\boldsymbol{u}}(t) & \geq \frac{\boldsymbol{E}_{\boldsymbol{u}}(t)}{R_{u} C_{u}}-\boldsymbol{P}_{\boldsymbol{u}}(t) \\
& -\frac{1}{R_{u} C_{u}} \sqrt{\boldsymbol{E}_{\boldsymbol{u}}(t)\left(\boldsymbol{E}_{\boldsymbol{u}}(t)-2 R_{u} C_{u} \boldsymbol{P}_{\boldsymbol{u}}(t)\right)}
\end{aligned}
$$

where relaxation was used with inequality that allows for higher losses than those corresponding to the used ultracapacitor power. The constraint (16) is convex, since $\boldsymbol{E}_{\boldsymbol{u}}(t)-2 R_{u} C_{u} \boldsymbol{P}_{\boldsymbol{u}}(t)$ is non-negative due to $(9 \mathrm{c})$, and it is obvious that at the optimum (16) will hold with equality. The constraints (9b)-(10c) will change to

$$
\boldsymbol{P}_{\boldsymbol{u}}(t) \geq i_{u \min } \sqrt{\frac{2 \boldsymbol{E}_{\boldsymbol{u}}(t) \boldsymbol{n}_{\boldsymbol{u}}}{C_{u}}}-R_{u} i_{u m i n}^{2} \boldsymbol{n}_{\boldsymbol{u}}
$$




$$
\begin{aligned}
& \boldsymbol{P}_{\boldsymbol{u}}(t) \leq i_{u \max } \sqrt{\frac{2 \boldsymbol{E}_{\boldsymbol{u}}(t) \boldsymbol{n}_{\boldsymbol{u}}}{C_{u}}}-\boldsymbol{B}_{\boldsymbol{u}}(t) \\
& \boldsymbol{P}_{\boldsymbol{u}}(t) \leq \frac{\boldsymbol{E}_{\boldsymbol{u}}(t)}{2 R_{u} C_{u}} \\
& \dot{\boldsymbol{E}}_{\boldsymbol{u}}(t)=-\left(\boldsymbol{P}_{\boldsymbol{u}}(t)+\boldsymbol{B}_{\boldsymbol{u}}(t)\right) \\
& \boldsymbol{E}_{\boldsymbol{u}}(t) \in\left[0, \frac{C_{u} u_{u \max }^{2}}{2} \boldsymbol{n}_{\boldsymbol{u}}\right] \\
& \boldsymbol{E}_{\boldsymbol{u}}\left(t_{0}\right)=\boldsymbol{E}_{\boldsymbol{u}}\left(t_{f}\right) .
\end{aligned}
$$

Similar convex modeling approach has been taken in [17], without introducing the new optimization variable $\boldsymbol{B}_{\boldsymbol{u}}(t)$.

Finally, the thermal constraints can be also modeled as convex by multiplying (12) with $\boldsymbol{n}_{\boldsymbol{u}}$ and introducing a variable change $\tilde{\boldsymbol{T}}_{\boldsymbol{u}}(t)=\boldsymbol{T}_{\boldsymbol{u}}(t) \boldsymbol{n}_{\boldsymbol{u}}$. Then, the constraints (12)-(14) will change to

$$
\begin{aligned}
& C_{T} R_{T} \dot{\tilde{\boldsymbol{T}}}_{\boldsymbol{u}}(t)=R_{T} \boldsymbol{B}_{\boldsymbol{u}}(t)+T_{a} \boldsymbol{n}_{\boldsymbol{u}}-\tilde{\boldsymbol{T}}_{\boldsymbol{u}}(t) \\
& \tilde{\boldsymbol{T}}_{\boldsymbol{u}}(t) \leq \boldsymbol{n}_{\boldsymbol{u}} T_{\text {umax }} \\
& \tilde{\boldsymbol{T}}_{\boldsymbol{u}}\left(t_{0}\right)=\tilde{\boldsymbol{T}}_{\boldsymbol{u}}\left(t_{f}\right) .
\end{aligned}
$$

\section{Convex battery model}

The battery open circuit voltage, illustrated in Fig. 4, can be approximated with a linear function

$$
u_{b}\left(\boldsymbol{s o c}_{\boldsymbol{b}}(t)\right)=d_{0} \boldsymbol{s o c}_{\boldsymbol{b}}(t)+d_{1}
$$

that gives good fit within the allowed SOC range. Then, the battery model can be written as convex by introducing a variable change

$$
\boldsymbol{E}_{\boldsymbol{b}}(t)=\frac{C_{b}\left(d_{0} \boldsymbol{s o c}_{\boldsymbol{b}}(t)+d_{1}\right)^{2} \boldsymbol{n}_{\boldsymbol{b}}}{2}
$$

where $C_{b}=2 Q_{b} / \bar{U}$ relates the battery capacity $Q_{b}$ with the nominal open circuit voltage $\bar{U}$ (dashed line in Fig. 4) and is used only to show resemblance with the ultracapacitor model. The remaining convex modeling steps are identical as those taken with the ultracapacitor.

\section{EXAMPLE OF POWERTRAIN SIZING}

This section gives an example of optimal powertrain sizing and control of a double buffer PHEV city bus.

\section{A. Problem setup}

The bus is equipped with a $220 \mathrm{~kW}$ EM and a $150 \mathrm{~kW}$ baseline EGU as in Fig. 3. The battery cells considered for the energy buffer have capacity of $45 \mathrm{Ah}$, while the ultracapacitor cells have capacity of $2000 \mathrm{~F}$. The remaining details of both cells are available online ${ }^{1}$. The operation is charge and temperature sustaining with free initial SOC and ambient temperature of $40^{\circ} \mathrm{C}$.

The bus line is as in Fig. 2 and two different charging scenarios are considered. The first scenario consists of seven chargers, placed on bus stops as in Fig. 2, which allow

\footnotetext{
${ }^{1}$ The battery cell is manufactured by Saft (http://www.saftbatteries.com) in lithium-ion technology and can be found under the name VL 45E. The ultracapacitor cell is manufactured by Maxwell (http://www.maxwell.com/) and can be found in the K2 series under the name BCAP2000 P270 (March 2012).
}

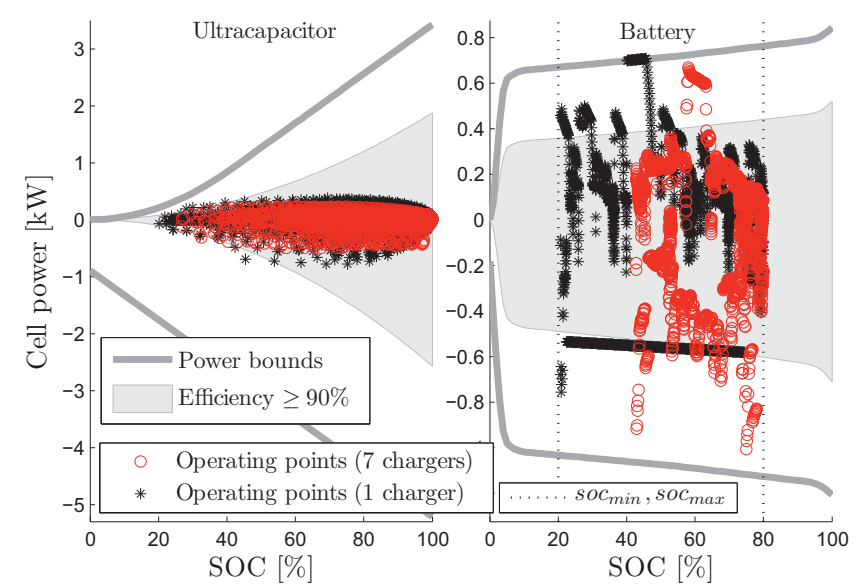

Fig. 5. Optimal operating points of the energy buffer. The ultracapacitor SOC is computed as $\boldsymbol{u}_{\boldsymbol{u}}(t) / u_{u \max }$.

the PHEV to charge with $P_{c \max }=200 \mathrm{~kW}$ for $10 \mathrm{~s}$ while standing still at the bus stop. The second scenario has only one charger which allows the PHEV to charge the battery for $10 \mathrm{~min}$ before starting the route, i.e. before $t=0$. In this case the vehicle is turned off during the charging period, i.e. there is no auxiliary load. The efficiency of all chargers is $92 \%$, but the charger power in the second scenario is left for the optimization to find it.

The convex problem is written in a time discrete form, the decision variables are scaled and a parser is used, CVX [18], to translate the problem in a general form of linear matrix inequalities required by the solver SeDuMi [19]. More details on the problem post-treatment can be found in [15].

\section{B. Optimization results}

The optimal results are obtained by iteratively solving the convex problem for 30 engine on-off power thresholds with grid size of $1 \mathrm{~kW}$. In each iteration, the computation time for solving the convex problem is $4-5 \mathrm{~min}$ on a standard $\mathrm{PC}^{2}$.

The optimal operating points of the energy buffer are given in Fig. 5, and the optimal state trajectories along the bus line are given in Fig. 6. The results show that the energy buffer is operated mainly with high efficiency and the ultracapacitor is utilized in rather low duty cycles, thus keeping its temperature safely below its upper limit of $65^{\circ} \mathrm{C}$.

The EGU size in both charging scenarios is kept at the minimum of $75 \mathrm{~kW}$. In the first scenario the EGU is kept on for $21 \%$ of the time, while in the $10 \mathrm{~min}$ charging scenario the bus is driven entirely on electric power. The optimal number of buffer cells is $\boldsymbol{n}_{\boldsymbol{u}}=403.2(0.82 \mathrm{kWh}), \boldsymbol{n}_{\boldsymbol{b}}=42.3$ (4.08 $\mathrm{kWh}$ usable) in the first scenario, and $\boldsymbol{n}_{\boldsymbol{u}}=244$ (0.49 kWh), $\boldsymbol{n}_{\boldsymbol{b}}=181.9$ (17.54kWh usable) in the second scenario. In terms of total cost the second scenario is better alternative (cost of $2.98 €$ vs. $5.53 €$ in the first scenario), if the schedule allows for the additional 10 min charging before starting the route. The charger, in this case, will need to have a power of at least $120 \mathrm{~kW}$.

\footnotetext{
${ }^{2} 2.67 \mathrm{GHz}$ dual core $\mathrm{CPU}$ and $4 \mathrm{~GB}$ RAM.
} 

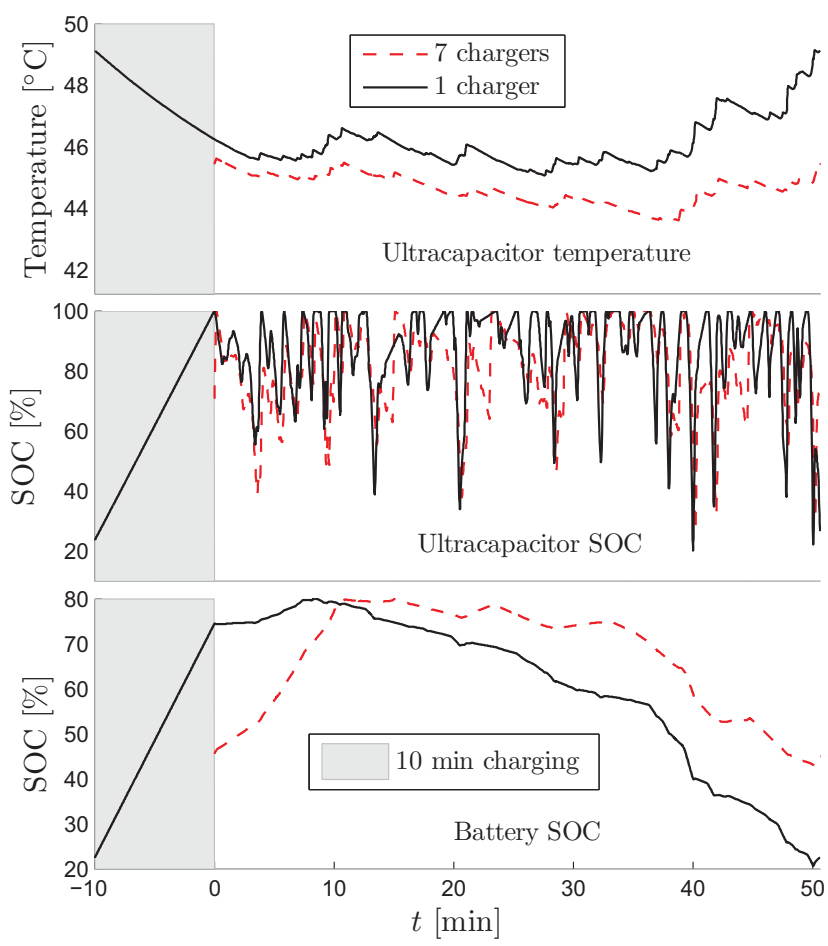

Fig. 6. Optimal temperature and SOC trajectories. The route starts at $t=0$.

\section{CONCLUSION}

This paper described modeling steps for simultaneous dimensioning and control of a PHEV bus using convex optimization. The method allows for thermal powertrain model and an example was given where an optimization problem with three continuous states can be solved in minutes.

Future studies may focus on extending the convex modeling approach to real time control of thermal powertrains and to simultaneous sizing and control of the cooling system for many vehicle components, including the passenger compartment.

\section{APPENDIX}

Denoting by $v(t)$ and $\alpha(t)$ the velocity and slope of the bus line, the angular velocity $\omega(t)$ and torque demanded on the shaft between the EM and the differential can be computed as

$$
\begin{aligned}
\omega(t) & =\frac{\gamma}{r} v(t), \quad m(\cdot)=m_{v b}+\boldsymbol{n}_{\boldsymbol{u}} m_{u}+\boldsymbol{n}_{\boldsymbol{b}} m_{b}+\boldsymbol{s} m_{E G U b} \\
\tau_{v}(\cdot) & =\left(I_{E M}+\frac{I}{\gamma^{2}}+m(\cdot) \frac{r^{2}}{\gamma^{2}}\right) \dot{\omega}(t)+\frac{\rho A_{f} c_{d} r^{3}}{2 \gamma^{3}} \omega^{2}(t) \\
& +\frac{g r}{\gamma} m(\cdot)\left(c_{r} \cos \alpha(t)+\sin \alpha(t)\right)
\end{aligned}
$$

where $g$ is gravitational acceleration, $\rho$ is air density, and the rest of the parameters are described in Table I. The model neglects the inertial effects of the EGU. The mass of the ultracapacitor and battery cell, $m_{u}, m_{b}$, have been included an additional $14 \%$ mass for packaging and circuitry.
TABLE I

PARAmeter VAlues.

\begin{tabular}{ll}
\hline Vehicle frontal area & $A_{f}=7.54 \mathrm{~m}^{2}$ \\
Aerodynamic drag coefficient & $c_{d}=0.7$ \\
Rolling resistance coefficient & $c_{r}=0.007$ \\
Wheel radius & $r=0.509 \mathrm{~m}$ \\
Final gear & $\gamma=4.7$ \\
Vehicle mass without buffer and EGU & $m_{v b}=13.7 \mathrm{t}$ \\
EM inertia & $I_{E M}=2.3 \mathrm{kgm}^{2}$ \\
Inertia of final gear and wheels & $I=41.8 \mathrm{kgm}^{2}$ \\
Sampling time & $1 \mathrm{~s}$ \\
\hline$P_{a}=7 \mathrm{~kW}, w_{f}=0.15 € / \mathrm{kWh}, w_{c}=0.1 € / \mathrm{kWh}, c_{u}=20.3 €$ \\
$c_{b}=80.4 €, c_{g}=6450 €, m_{E G U b}=800 \mathrm{~kg}$
\end{tabular}

\section{REFERENCES}

[1] L. Guzzella and A. Sciarretta, Vehicle propulsion systems, introduction to modeling and optimization, 2nd ed. Berlin, Heidelberg: Springer, 2007.

[2] "AutoTram: Transport system of the future," Fraunhofer Institute for Transportation and Infrastructure Systems IVI, Tech. Rep., 2010.

[3] M. Johansson and O. Olsson, "Feasibility study of dual-mode buses in Gothenburg's public transport," Master's thesis, Chalmers University of Technology, Gothenburg, Sweden, 2011.

[4] R. Bedell, B. Westerlund, and P. Åstrand, "First results from field testing of fast charged hybrid buses in Umea, sweden," in EEVC, Brussels, Belgium, October 26-28 2011.

[5] "On-line electric vehicle," Korea Advanced Institute of Science and Technology (KAIST), Tech. Rep., 2009.

[6] A. Burke, "Ultracapacitor technologies and application in hybrid and electric vehicles," International Journal of Energy Research, vol. 34, no. 2, pp. 133-151, 2010.

[7] L. Johannesson, S. Pettersson, and B. Egardt, "Predictive energy management of a 4QT series-parallel hybrid electric bus," Control Engineering Practice, vol. 17, no. 12, pp. 1440-1453, December 2009.

[8] T. C. Moore, "HEV control strategy: Implications of performance criteria, system configuration and design, and component selection," in Proceedings of the American Control Conference, Albuquerque, New Mexico, June 1997.

[9] R. Bellman, Dynamic Programming. New Jersey: Princeton Univ Pr, June 1957.

[10] U. Zoelch and D. Schroeder, "Dynamic optimization method for design and rating of the components of a hybrid vehicle," International Journal of Vehicle Design, vol. 19, no. 1, pp. 1-13, 1998.

[11] M. Kim and H. Peng, "Power management and design optimization of fuel cell/battery hybrid vehicles," Journal of Power Sources, vol. 165, no. 2, pp. 819-832, 2007.

[12] O. Sundström, L. Guzzella, and P. Soltic, "Torque-assist hybrid electric powertrain sizing: From optimal control towards a sizing law," IEEE Transactions on Control Systems Technology, vol. 18, no. 4, pp. 837849 , July 2010

[13] S. J. Moura, D. S. Callaway, H. K. Fathy, and J. L. Stein, "Tradeoffs between battery energy capacity and stochastic optimal power management in plug-in hybrid electric vehicles," Journal of Power Sources, vol. 195, no. 9, pp. 2979-2988, 2010.

[14] N. Murgovski, J. Sjöberg, and J. Fredriksson, "A methodology and a tool for evaluating hybrid electric powertrain configurations," Int. J. Electric and Hybrid Vehicles, vol. 3, no. 3, pp. 219-245, 2011.

[15] N. Murgovski, L. Johannesson, J. Sjöberg, and B. Egardt, "Component sizing of a plug-in hybrid electric powertrain via convex optimization," Journal of Mechatronics, vol. 22, no. 1, pp. 106-120, 2012.

[16] S. Boyd and L. Vandenberghe, Convex Optimization. Cambridge University Press, 2004.

[17] N. Murgovski, L. Johannesson, and J. Sjöberg, "Convex modeling of energy buffers in power control applications," in IFAC Workshop on Engine and Powertrain Control, Simulation and Modeling (ECOSM), Rueil-Malmaison, Paris, France, October 23-25 2012.

[18] M. Grant and S. Boyd, "CVX: Matlab software for disciplined convex programming, version 1.21," http://cvxr.com/cvx, May 2010.

[19] Y. Labit, D. Peaucelle, and D. Henrion, "SeDuMi interface 1.02: a tool for solving LMI problems with SeDuMi," IEEE International Symposium on Computer Aided Control System Design Proceedings, pp. 272-277, September 2002. 